\title{
Optimization of the NZ-1 labeling technique for the application to 3D structure analysis
}

\author{
R. Aruga ${ }^{1}$, R. Tamura-Sakaguchi ${ }^{1}$, M. Hirose ${ }^{2}$, T. Ekimoto ${ }^{1}$, R. Oi ${ }^{1}$, M. K. Kaneko ${ }^{3}$, Y. Kato ${ }^{3,4}$, M. Ikeguchi ${ }^{1,5}$, \\ K. Iwasaki ${ }^{6}$, T. Nogi ${ }^{1}$ \\ ${ }^{I}$ Graduate School of Medical Life Science, Yokohama City University, Japan, \\ ${ }^{2}$ Institute for Protein Research, Osaka University, Japan, \\ ${ }^{3}$ Department of Antibody Drug Development, Tohoku University Graduate School of Medicine, Japan, \\ ${ }^{4}$ New Industry Creation Hatchery Center, Tohoku University, Japan, \\ ${ }^{5}$ Center for Computational Science, RIKEN, Japan, and \\ ${ }^{6}$ Life Science Center for Survival Dynamics, Tsukuba Advanced Research Alliance (TARA), University of Tsukuba, Japan. \\ w205402b@yokohama-cu.ac.jp
}

Antibody labeling has become a useful tool for determining three-dimensional (3D) structures of protein molecules and their complexes. Antibody fragments such as antigen-binding fragments (Fabs) serve as crystallization chaperones to promote lattice formation in X-ray crystallography. On the other hand, Fabs can be used as fiducial marks to aid the particle picking and alignment in cryo-electron microscopy (EM), which is difficult to apply to small protein molecules. However, establishing antibodies that bind stably to their respective targets is a prerequisite for utilizing the antibody labeling. To expand its applicability, we have developed an alternative strategy by inserting an exogenous epitope into targets and subsequently preparing complexes with antibody fragments. Specifically, we utilized a monoclonal antibody NZ-1, which has been established by immunizing rat with a 14-residue peptide segment (PA14) from human podoplanin. It has been shown that a 12-residue peptide segment (PA12) lacking two N-terminal residues of PA14 binds to NZ-1 with an extremely high affinity.$^{[1]}$ In addition, a previous crystallographic analysis of the NZ-1 Fab complexed with the PA14 peptide has revealed that the PA12 part adopts a bent loop-like conformation within the antigen-binding pocket of NZ-1. Based on these observations, we first attempted to develop the NZ-1 labeling technique using the PA12 tag. We inserted the PA12 tag into the PDZ tandem (PDZ-N and PDZ-C) located in the periplasmic region of intramembrane protease. In fact, the PA12-inserted PDZ tandem formed a stable complex with the NZ-1 Fab. ${ }^{[2]}$ However, our structural analysis also showed that the complex formation through the inserted PA12 tag inevitably caused structural changes around the insertion site on the target. Therefore, we next attempted to utilize the PA14 tag, instead of PA12, which contains Glu-Gly residues upstream of PA12. We expected that the two additional residues could serve as a buffer region to tolerate structural changes on the target because they are flexible in the above-mentioned crystal structure of the NZ-1 Fab complexed with the PA14 peptide. As a result, the PA14-inserted PDZ tandem also produced co-crystals with the NZ-1 Fab, where the complex formation had less impact on the folding of the target PDZ domains as compared to the NZ-1 labeling with the PA12 tag. ${ }^{[3]}$ In addition, molecular-dynamics (MD) simulations have also suggested that PA14-inserted PDZ domains stably bind to the NZ-1 Fab with no significant structural changes. To demonstrate that our improved NZ-1 labeling technique could be applied to EM analysis, we also performed negative stain EM on the NZ-1-labeled full-length intramembrane protease. We obtained 3D models of the complex and succeeded in approximating the spatial arrangement of the PDZ domains based on the docking mode of the NZ-1 Fab.

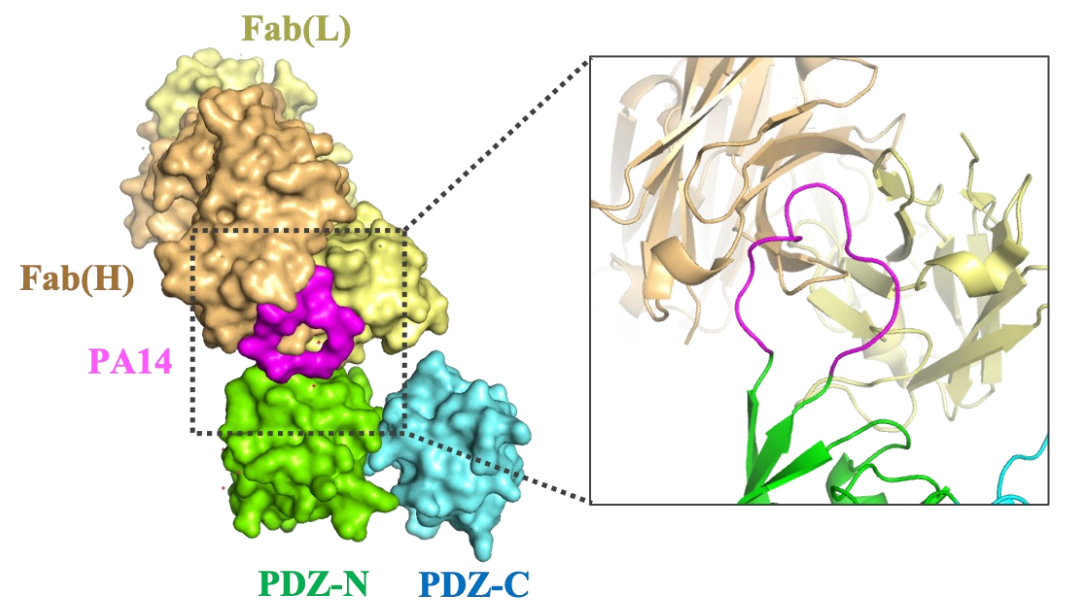

Figure 1. Crystal structure of PA14-inserted PDZ tandem complexed with the NZ-1 Fab. Overall structure (left) and close-up view around the antigen-binding site (right). 
[1] Fujii, Y., Kaneko, M., Neyazaki, M., Nogi, T., Kato, Y. \& Takagi, J. (2014). Protein Expr. Purif. 95, 240-247.

[2] Tamura, R., Oi, R., Akashi, S., Kaneko, M., Kato, Y. \& Nogi, T. (2019). Protein Sci. 28, 823-836.

[3] Tamura-Sakaguchi, R., Aruga, R., Hirose, M., Ekimoto, T., Miyake, T., Hizukuri, Y., Oi, R., K.Kaneko, M., Kato, Y., Akiyama, Y., Ikeguchi, M., Iwasaki, K. \& Nogi, T. (2021). Acta Cryst. D77, 645-662

Keywords : antibody labeling ; epitope insertion ; antibody-assisted structural analysis ; electron microscopy ; protein crystallography 